\title{
Critical dimension of biperiodic gratings determined by spectral ellipsometry and Mueller matrix polarimetry
}

\author{
M. Foldyna ${ }^{1, a}$, A. De Martino ${ }^{1}$, E. Garcia-Caurel ${ }^{1}$, R. Ossikovski ${ }^{1}$, C. Licitra ${ }^{2}$, F. Bertin ${ }^{2}$, \\ K. Postava ${ }^{3}$, and B. Drevillon ${ }^{1}$ \\ 1 Laboratoire de Physique des Interfaces et Couches Minces, UMR 7647, École Polytechnique, 91128 Palaiseau Cedex, France \\ 2 CEA LETI - MINATEC, 17 rue des Martyrs, 38054 Grenoble Cedex 9, France \\ 3 Department of Physics, Technical University of Ostrava, 17. listopadu 15, 70833 Ostrava, Czech Republic
}

Received: 12 November 2007 / Received in final form: 28 January 2008 / Accepted: 2nd April 2008 Published online: 4 June 2008 - (C) EDP Sciences

\begin{abstract}
We characterized two samples consisting of photoresist layers on silicon with square arrays of square holes by spectroscopic ellipsometry (SE) and Mueller matrix polarimetry (MMP). Hole lateral dimensions and depths were determined by fitting either SE data taken in conventional planar geometry or MMP data in general conical diffraction configurations. A method for objective determination of the optimal measurement conditions based on sensitivity and parameter correlations is presented. When applied to MMP, this approach showed that for one of the samples the optimal incidence angle was $45^{\circ}$, much below the widely used $70^{\circ}$ value. The robustness of the dimensional characterisation based on MMP is demonstrated by the high stability of the results provided by separated fits of the data taken at different azimuthal angles.
\end{abstract}

PACS. 42.79.Dj Gratings - 07.60.Fs Polarimeters and ellipsometers - 95.75.Hi Polarimetry - 42.62.Eh Metrological applications; optical frequency synthesizers for precision spectroscopy

\section{Introduction}

Critical dimension (CD) monitoring by optical methods evolved into the development of fast, non-destructive characterization tools with a low cost of ownership. Nowadays, spectral ellipsometry (also called "scatterometry") is already widely used in semiconductor industry for dimensional characterization of mono-, and to a lesser extent, bi-periodic structures [1]. In the last few years, spectrally resolved Mueller matrix polarimetry (MMP) has also demonstrated a great potential in this field [2,3].

The characterization procedure is based on the solution of an inverse problem by fitting the data measured in specular reflection with a multiparameter model. Its overall performance, in terms of reproducibility and accuracy, heavily depends on the quality of the measured data and its sensitivity to the fitting parameters. Sensitivity can be improved by optimising the experimental configuration, as it has been emphasized in previous works [4]. The importance of proper configuration selection is even more important for Mueller polarimetric measurements than for conventional SE, owing to the additional information provided by complete Mueller matrices when not only

\footnotetext{
a e-mail: marfol@gmail.com
}

incidence, but also azimuthal angle is varied, i.e. when the most general conical diffraction configuration is considered.

A recent study of the dependence of parameter variances and correlations [5] for MMP in conical diffraction showed that Mueller matrices taken for different azimuthal angles can lead to different precision of the estimated parameters. Moreover, it was shown that some configurations have significantly smaller correlations between parameters, which has a positive impact on the fit.

Characterization of biperiodic samples significantly increases computation requirements on modeling. These requirements are the main slowdown of current progress in modeling $2 \mathrm{D}$ gratings. Therefore any possible insight into the issues related to decreasing the calculation time while keeping the same precision would be welcome. In this work we show that this problem can be addressed by two basic methods: (a) optimization of the numerical calculations either by some physical insight, advanced mathematical methods or simply by introducing parallelism, or (b) by a proper choice of the experimental configurations allowing to decrease number of iterations during fitting and possibly also the number of spectral points, without losing certainty of fitted results. Particular methods used to calculate diffraction problem are presented in Section 4. 
We propose to choose azimuthal and incidence angles with respect to theoretical calculations revealing combination of small correlations between parameters and small parameters estimation errors. Considering only correlations between parameters would not lead to the optimal choice as it does not reflect parameter sensitivities to the data measured at different incidence and azimuthal angles. In Section 5 we show how to use "virtual measurements" (modelled data with white noise) to obtain correlations between parameters and ideal statistical errors of the parameters. This information is then used to suggest measurement configuration suitable for given type of grating.

In Section 6 we present measured data and fits of $2 \mathrm{D}$ periodic square holes produced by UV lithography in photoresist deposited on a silicon substrate (see Figs. 2 and 3). Consistency of our model is checked by fitting independently wide range of incident and azimuthal angles separately. Note that data taken at different conical configurations brings independent information and as such it can be further statistically evaluated. That is, realistic error of the fitted parameters can be estimated from the values obtained from different experimental configurations dispersed around an average.

\section{Experimental setup}

The grating samples were characterized by Horiba Jobin-Yvon Spectroscopic Phase Modulated Ellipsometer (UVISEL) in planar diffraction geometry in the spectral range from $0.8 \mathrm{eV}$ to $4.7 \mathrm{eV}$. The UVISEL in the standard PMSA configuration incorporates a photo-elastic device to modulate the illumination beam polarization and a linear analyser after the sample. The measurement itself involves a lock-in processing of the detector signal at both the photo-elastic driving frequency and its first harmonic and to provide two quantities, $I_{s}$ and $I_{c}$. With the modulator at $45^{\circ}$ and the analyzer at $0^{\circ}$ these quantities are nothing else but the following elements of normalized Mueller matrix:

$$
\begin{aligned}
& I_{s}=\sin 2 \psi \sin \Delta=M_{43}, \\
& I_{c}=\sin 2 \psi \cos \Delta=M_{33},
\end{aligned}
$$

where $\psi$ and $\Delta$ are ellipsometric angles [6].

In its current configuration, the ellipsometer does not have access to off-diagonal block elements of the Mueller matrix. Moreover, on this setup the incidence angle was fixed at $70^{\circ}$ and we could not vary azimuthal angle with sufficient accuracy. Therefore, with the UVISEL the sample was measured only in planar configuration, with the plane of incidence parallel to the edge of square holes. This azimuth could be found precisely by geometric alignment of higher diffraction orders into one plane.

Measurements of the samples in the most general configurations (i.e. with various incidence and azimuthal angles) were done by Horiba Jobin-Yvon Liquid Crystal Mueller Matrix Polarimeter (MM16) operating in the visible range (450-825 nm). A schematic figure of the MM16

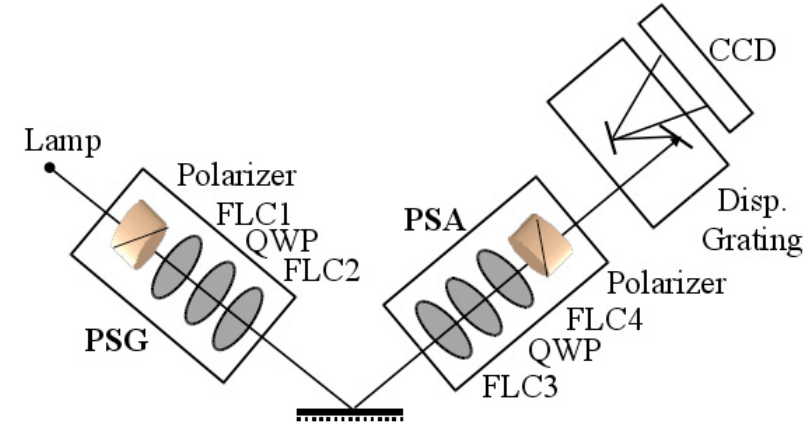

Fig. 1. Scheme of the Liquid Crystal Mueller Matrix Polarimeter in reflection configuration. Light source is halogen lamp and blue LED providing together unpolarized light from 425 to $850 \mathrm{~nm}$. The Polarization State Generator (PSG) and Analyzer (PSA) are each composed of an linear polarizer, quartz quarter-wave plate (QWP), and two ferroelectric liquid crystal devices (FLC).

polarimeter in reflection configuration is shown in Figure 1. The Polarization State Generator (PSG) consists of a linear polarizer, a quartz retardation plate QWP), and two ferroelectric liquid crystal devices (FLCs), each of which can be switched between two different states. As a result, the PSG can generate four different polarization states for the illumination beam. The PSA comprises the same elements in reverse order, and is used to analyse the polarization of the emerging beam over another set of four different polarization states. Finally, the polarimeter subsequently measures a set of 16 raw spectra, each of which is taken with a known state of the PSG and the PSA.

This instrument is calibrated by using the Eigenvalue Calibration Method [7], which is general and selfconsistent method for the calibration of polarization modulators, polarimeters and Mueller-matrix ellipsometers. The aim of calibration is to find modulation and analysis matrices $\mathrm{W}$ and $\mathrm{A}$ which describe the polarization states actually generated by the PSG and PSA. These matrices are obviously wavelength dependent, and for each wavelength the Mueller matrix $M_{\lambda}$ of the sample is deduced from the 16 raw intensities $\mathrm{B}_{\lambda}$ as:

$$
\mathrm{M}_{\lambda}=\mathrm{A}_{\lambda}^{-1} \mathrm{~B}_{\lambda} \mathrm{W}_{\lambda}^{-1} \text {. }
$$

The PSG and PSA are designed in order to make the modulation and analysis matrices as close as possible to unitary matrices, by minimizing their condition numbers over the measured spectrum. This design is aimed at minimising the noise in the final Mueller matrices $M_{\lambda}$ for a given additive noise in the raw intensity measurements $\mathrm{B}_{\lambda}$. Moreover, with this optimised design, it can be shown [8] that the noise is equally distributed among the various elements of $\mathrm{M}_{\lambda}$, as all raw data elements do contribute with comparable weights in each element of the final Mueller matrix. More detailed description of MM16 polarimeter including the method of calibration can be found in [9].

In contrast with our UVISEL, which was operated at fixed $70^{\circ}$ incidence and zero azimuthal angle, the MM16 allowed easy and accurate variation of both incidence and 

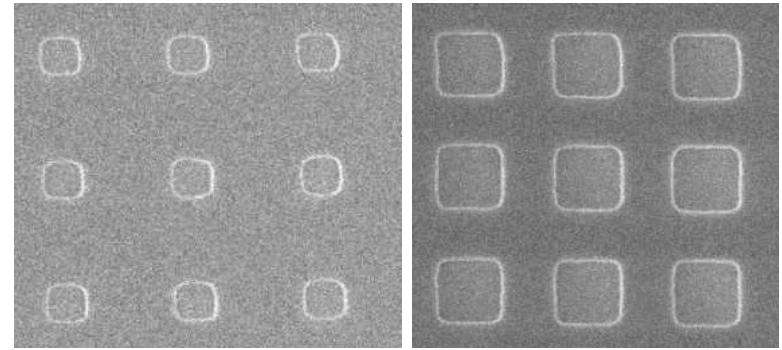

Fig. 2. Electron microscope images of $250 \times 250 \mathrm{~nm}$ and 500 $\times 500 \mathrm{~nm}$ holes in $400 \mathrm{~nm}$ thick photoresist. Periods in both directions are $1 \mu \mathrm{m}$.

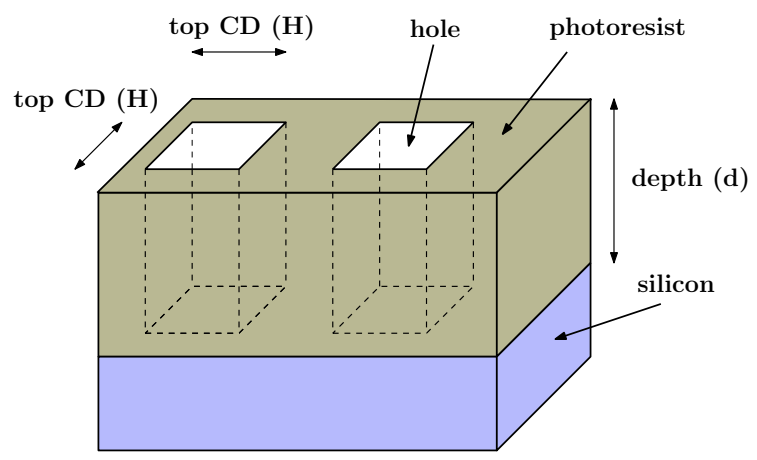

Fig. 3. Schematic picture of the grating profile. Critical dimension of the grating is hole width denoted by $H$. Depth of the hole in resist layer is denoted by $d$.

azimuth, the former from $45^{\circ}$ to $70^{\circ}$ (limited by the spot size) and the latter over all possible values from $0^{\circ}$ to $360^{\circ}$. In this work, the azimuth was varied from $-90^{\circ}$ to $90^{\circ}$ in steps of $5^{\circ}$.

\section{Sample characteristics}

Samples of $2 \mathrm{D}$ periodic gratings were prepared in CEA - LETI by using UV lithography process. These gratings consisted of square arrays of square holes in $400 \mathrm{~nm}$ thick layers of photoresist deposited on crystalline silicon. Grating period was $1 \mu \mathrm{m}$ in both directions, while nominal dimensions of holes were $250 \times 250$ and $500 \times 500 \mathrm{~nm}$. Critical dimension (CD) to be determined by fitting the data are hole width and depth. Electron microscopy images and schematic picture of the sample are shown in Figures 2 and 3 .

Optical function of photoresist was determined from ellipsometric measurement on a non-patterned part of the sample and material parameters of crystalline silicon were taken from Palik [10]. Photoresist is transparent in lower energy spectrum, but exhibits absorption in the ultraviolet part of the spectrum. In the selected spectral range $1-3.5 \mathrm{eV}$ optical function of photoresist was modeled by the Tauc-Lorentz model [11], which imaginary part of the complex dielectric function is given by:

$$
\varepsilon_{2}(E)=\left\{\begin{array}{cc}
\frac{1}{E} \frac{A E_{0} C\left(E-E_{g}\right)^{2}}{\left(E^{2}-E_{0}^{2}\right)^{2}+C^{2} E^{2}} & \left(E>E_{g}\right) \\
0 & \left(E \leq E_{g}\right)
\end{array} .\right.
$$

The real part of the dielectric function is determined in a closed form from $\varepsilon_{2}$ using Kramers-Krönig integration [11]. The model dielectric function employs five fitting parameters: the non-dispersive term $\varepsilon_{\infty}=1.80$, the band gap energy $E_{g}=1.26$, the amplitude $A=5.17$, the Lorentz resonant frequency $E_{0}=6.53$, and the broadening parameter $C=0.11$.

Reference values of CDs were taken from fit of the ellipsometric data obtained by UVISEL for spectral range from $1 \mathrm{eV}$ to $3.5 \mathrm{eV}$ and typical incidence angle $70^{\circ}$. The wider spectral range of the ellipsometer provided better sensitivity to the grating depth, when compared to the polarimeter.

\section{Optical modeling}

Optical response of grating is modeled by a $2 \mathrm{D}$ version of standard rigorous coupled-waves method (RCWM) [12] based on a projection of Maxwell equations to a Fourier basis and reducing them to a set of ordinary differential equations.

Implementation is extended by using factorization of permittivity tensor based on the Li inverse rules [13] and boundary conditions on each interface are treated by scattering matrix (S-matrix) algorithm [14]. RCWA with these two extensions is nowadays standard approach to the $1 \mathrm{D}$ lamellar and 2D dot gratings. It leads to very fast calculations, where only relatively small number of the Fourier harmonics is necessary and also avoids numerical instability otherwise appearing in the cases of deeper gratings. Further, eigenvalue problem was tweaked the way, that only about $50-75 \%$ of modes were used depending on their significance. This alone allowed to further decrease calculation time by about a factor of two, while the precision of the calculations did not exhibit significant changes.

The code provides the complex Jones matrix of zero diffraction order of the grating

$$
\mathrm{J}=\left[\begin{array}{ll}
J_{11} & J_{12} \\
J_{21} & J_{22}
\end{array}\right] .
$$

The corresponding Mueller matrix $\mathrm{M}$ can be calculated from the Jones matrix $J$ and expressed in the form of four block matrices:

$$
\mathrm{M}=\left[\begin{array}{llll}
M_{11} & M_{12} & M_{13} & M_{14} \\
M_{21} & M_{22} & M_{23} & M_{24} \\
M_{31} & M_{32} & M_{33} & M_{34} \\
M_{41} & M_{42} & M_{43} & M_{44}
\end{array}\right]=\left[\begin{array}{ll}
\mathrm{B}_{11} & \mathrm{~B}_{12} \\
\mathrm{~B}_{21} & \mathrm{~B}_{22}
\end{array}\right],
$$

where the expressions for the block matrices follows:

$$
\begin{aligned}
\mathrm{B}_{11} & =\left[\begin{array}{cc}
I^{2} & I^{2}-J_{12}^{2}-J_{22}^{2} \\
I^{2}-J_{21}^{2}-J_{22}^{2} & I^{2}-J_{12}^{2}-J_{21}^{2}
\end{array}\right], \\
\mathrm{B}_{12} & =\left[\begin{array}{ll}
\Re\left(P_{1112}+P_{2122}\right) & -\Im\left(P_{1112}+P_{2122}\right) \\
\Re\left(P_{1112}-P_{2122}\right) & -\Im\left(P_{1112}-P_{2122}\right)
\end{array}\right], \\
\mathrm{B}_{21} & =\left[\begin{array}{ll}
\Re\left(P_{1121}+P_{1222}\right) & \Re\left(P_{1121}-P_{1222}\right) \\
\Im\left(P_{1121}+P_{1222}\right) & \Im\left(P_{1121}-P_{1222}\right)
\end{array}\right], \\
\mathrm{B}_{22} & =\left[\begin{array}{ll}
\Re\left(P_{1122}+P_{1221}\right) & -\Im\left(P_{1122}-P_{1221}\right) \\
\Im\left(P_{1122}+P_{1221}\right) & \Re\left(P_{1122}-P_{1221}\right)
\end{array}\right] .
\end{aligned}
$$


Here $J_{i j}^{2}$ denotes $\left|J_{i j}\right|^{2}, I^{2}=\frac{1}{2}\left(J_{11}^{2}+J_{12}^{2}+J_{21}^{2}+J_{22}^{2}\right)$, and $P_{i j k l}=J_{i j}^{*} J_{k l}$. As the absolute reflectivity of the system is unknown, sample is characterized by a normalized Mueller matrix $\mathrm{M}_{n}$, which is related to $\mathrm{M}$ by the simple relation $\mathrm{M}_{n}=I^{-2} \mathrm{M}$.

Block matrix $\mathrm{B}_{11}$ from (7) contains information about reflectivities, where in our case of normalized Mueller matrices the total reflectivity of the system is unknown. Block $\mathrm{B}_{22}$ in (10) is connected to the ellipsometric parameters, where for isotropic samples the following relations hold: $I_{s}=M_{43}=-M_{34}, I_{c}=M_{33}=M_{44}$. Ellipsometric parameters $I_{s}$ and $I_{c}$ can be written by using ellipsometric angles $\psi$ and $\Delta$ as in (1) and (2), respectively. Off-diagonal blocks $\mathrm{B}_{12}$ and $\mathrm{B}_{21}$ from (8) and (9) are adherent to an anisotropic behavior of gratings. In our case $\mathrm{B}_{12}$ and $\mathrm{B}_{21}$ are zeros when grating is in planar configuration or rotated by azimuthal angle of $45^{\circ}$ (due to the symmetry of square profiles of holes). Note that our gratings are symmetrical to the azimuthal angle rotation by $90^{\circ}$, therefore two equal planar configurations exist.

\section{Fitting and optimal measurement configuration}

Sensitivity of fitted parameters to the experimental data and correlations between them can be studied by using rigorous modeled data with applied white noise of amplitude $\sqrt{3} \sigma$, where $\sigma$ is estimated precision of experimental data. In our work $\sigma$ is chosen equal to 0.01 , which is close to the experimental precision of our measurement setup.

Used fitting parameters of the model are only two critical dimension $(H)$ and grating depth $(d)$. Merit function relating our model to experimental data used in this section is defined as

$f^{\text {merit }}(H, d)=\frac{1}{15 K-3} \sum_{k=1}^{K} \sum_{i, j} \frac{\left|M_{i j k}^{\text {meas }}-M_{i j k}^{\text {calc }}(H, d)\right|^{2}}{\sigma_{i j k}^{2}}$,

where $k$ denotes the spectral point from the total number $K$, indexes $i, j$ denote all Mueller matrix elements except $M_{11}$ and experimental errors $\sigma_{i j k}$ are taken all equal to $\sigma=0.01$. This assumption of uniform noise over all values of indexes $i, j, k$ would be rigorously justified only for ideal situations, with a perfectly adequate model and a purely statistical noise with no systematic errors. As discussed below, in reality this is not the case, but this assumption is adequate enough to derive the optimal configurations from "virtual experiments" based only on simulations and on the standard evaluation of the parameter variances and correlations outlined below.

Considering a set of experimental data $M_{r}$ (in our case these would be the $M_{i j k}$ Mueller matrix elements, with the three indexes $i, j$ and $k$ lumped into a single index $r$ ) and a set of parameters $\beta_{s}$ (in our case $H$ and $d$ ) we define the (rectangular) Jacobian matrix $\mathrm{J}$ by

$$
J_{r s}=\frac{\partial M_{r}}{\partial \beta_{s}} .
$$

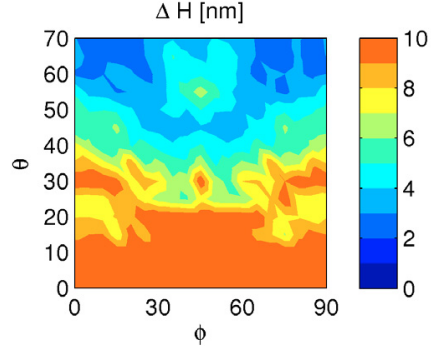

(a) $250 \mathrm{~nm}$ holes

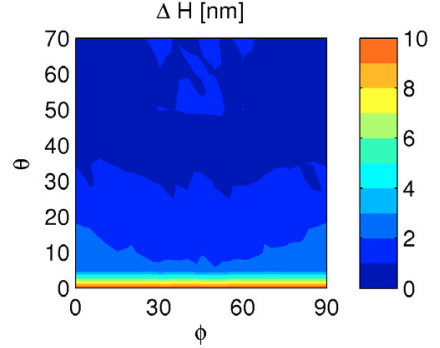

(b) $500 \mathrm{~nm}$ holes
Fig. 4. (Color online) Error estimation $\Delta H$ of CD, plotted for range of incidence $\theta$ and azimuthal angles $\phi$. Errors are noted by different colours with values found in the bar on the right, values are in nanometers. Figure is obtained from simulated data calculated every $5^{\circ}$ of incidence and azimuthal angle.

The variance-covariance matrix of the parameters is then given by $[15]$ :

$$
\mathrm{V}=\sigma^{2}\left(\mathrm{~J}^{T} \mathrm{~J}\right)^{-1}
$$

The diagonal elements $V_{i i}$ correspond to the squares of standard deviations of parameters. The correlations $C_{i j}$ between different parameters can be obtained from the off-diagonal elements of $\mathrm{V}$ (the parameter covariances) as:

$$
C_{i j}=\frac{V_{i j}}{\sqrt{V_{i i} V_{j j}}} .
$$

In principle, these formulas are valid only in the ideal case of purely statistical additive noise, not necessarily Gaussian but with a uniform and finite RMS value $\sigma$. Moreover, the noises affecting different experimental data are supposed to be fully decorrelated. Even though these assumptions are not fulfilled in our case (as in most real situations) this procedure is the only one which can be reasonably implemented to optimize the measurement configurations and it provides useful results, as shown below.

The variance-covariance matrices and corresponding correlation matrices are evaluated for the range $0-70^{\circ}$ of incidence angles $\theta$ and $0-90^{\circ}$ of azimuthal angles $\phi$ with the step of $5^{\circ}$ in both. The modelled experimental data are normalized Mueller matrices in the $450-825 \mathrm{~nm}$ spectral range.

The standard errors of $H$ corresponding to $95 \%$ confidence interval are plotted in Figure 4 for both grating samples. From the figure one can immediately conclude that the grating with $500 \mathrm{~nm}$ holes has much better determined values of $H$ and $d$ for all possible configurations. The reason for this is the big difference between fill factors of the two gratings $(1 / 4$ and $1 / 16)$, together with the fact that $250 \mathrm{~nm}$ holes are smaller than all the wavelengths of incident light. Nevertheless, the trends are the same in both figures, showing decreasing errors with increasing angles of incidence. Higher values of errors for azimuthal angles around $45^{\circ}$ and incidence angles around $70^{\circ}$ suggest that experimental configurations close to planar configuration should be preferred.

The errors in determination of grating depth $d$ are plotted in Figure 5 for both samples. The depth is very 


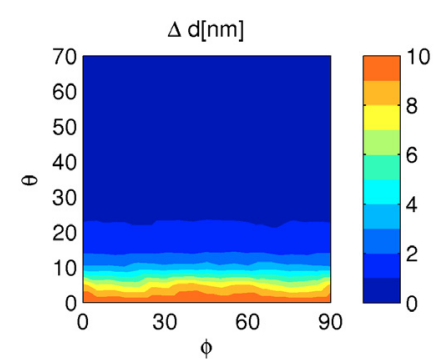

(a) $250 \mathrm{~nm}$ holes

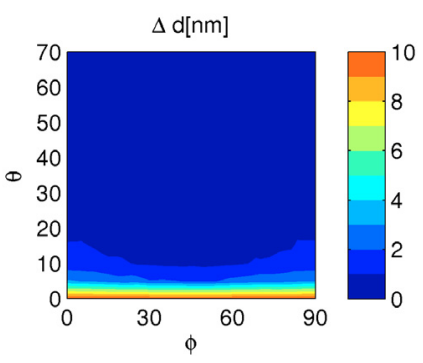

(b) $500 \mathrm{~nm}$ holes
Fig. 5. (Color online) Error estimation $\Delta d$ of the grating depth, plotted for range of incidence and azimuthal angles. Statistical errors are expressed in nanometers.

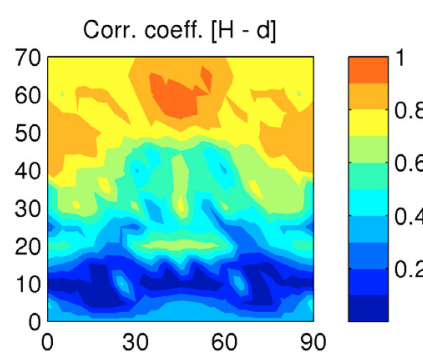

(a) $250 \mathrm{~nm}$ holes

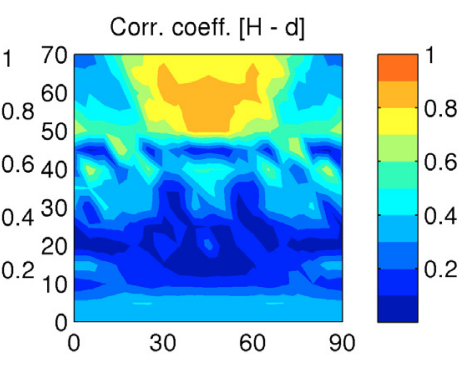

(b) $500 \mathrm{~nm}$ holes
Fig. 6. (Color online) Correlation coefficient between fitted parameters $H$ and $d$ plotted for range of incidence and azimuthal angles. Values for different colours can be found in the bar on the right. Figure is plotted from data calculated every $5^{\circ}$ of incidence and azimuthal angle.

precisely determined except incidence angles close to normal incidence. Therefore, preferences are the same as in the case of $\Delta H$ towards the higher angles of incidence.

A very interesting situation can be observed at Figure 6 , where correlation coefficient between $H$ and $d$ is plotted. The plot for $250 \mathrm{~nm}$ holes shows smaller correlations between parameters for smaller incidence angles. Though, when one shall decide for more advantageous configuration, errors in the parameters are more important. Smaller correlation would lead to a smoother fitting, but the precision of the fitted result would not be that good. Therefore, we look for the configurations with relatively small errors with not so bad correlations. In the case of the sample with $250 \mathrm{~nm}$ holes an advantageous configuration is at incidence angle as big as $70^{\circ}$ and azimuthal angle close to $0^{\circ}$ (planar configuration). At that configuration the errors of fitted parameters are the smallest and correlation coefficient is under 0.8 .

Sample with $500 \mathrm{~nm}$ holes features a surprising result. On one hand, one can naturally think of the same configuration as for the case with $250 \mathrm{~nm}$ holes with incidence angle of $70^{\circ}$ and azimuthal angle close to $0^{\circ}$. Surprisingly though, the better choice is incidence angle of $45^{\circ}$ for almost any choice of azimuthal angle. Errors in fitted parameters are about the same as for high incidence angles, while correlation between parameters is much bet- ter, in some cases under 0.2 . This is nice example of the fact that higher incidence angle is not always better and it also shows usefulness of presented approach to choose advantageous experimental configuration.

\section{Results and discussions}

Based on the results reported above, polarimetric measurements of the $250 \times 250 \mathrm{~nm}$ sample were accomplished by MM16 for incidence angle of $70^{\circ}$, while azimuthal angles was varied from $-90^{\circ}$ to $90^{\circ}$ in steps of $5^{\circ}$. Polarimeter provides normalized Mueller matrices for the spectral range of wavelengths from 450 to $825 \mathrm{~nm}$. The sample with $500 \times 500 \mathrm{~nm}$ holes was measured with the same azimuths, but with incidences varying from $45^{\circ}$ to $70^{\circ}$ in steps of $5^{\circ}$. In this work we fitted the data by using nonlinear Levenberg-Marquardt method [15], which typically converges very fast to minimum if suitable initial conditions are chosen.

The results of the fits of $I_{s}$ and $I_{c}$ data measured for both samples in planar configuration at incidence angle of $70^{\circ}$ are plotted in Figure 7. Measured data are denoted by squares and rounds in the figure. Curves of measured data for the $250 \mathrm{~nm}$ holes sample are very smooth and simple and the quality of the fit is better than for $500 \mathrm{~nm}$ holes. During fitting only 50 points were used as inclusion of more points increases significantly computation time and does not provide much more information. Fitted parameters of two samples give the values of CDs 250.3 and $497.4 \mathrm{~nm}$ and depths 393.5 and $406.1 \mathrm{~nm}$, which will be compared with the results of the fits of polarimetric data. To compare the ellipsometric fit with polarimetric ones on the sample with $250 \mathrm{~nm}$ holes, data for all azimuthal angles (each one separately) with 30 spectral points were fitted and results are plotted in Figure 8. Spectral points are chosen equidistantly from the wavelength range of 450-850 nm shown in Figure 9. Zero azimuthal angle is set to the planar configuration, with the incidence plane parallel to hole walls. Incidence angle is $70^{\circ}$ in all cases. Comparison with the values from ellipsometric fit gives very good correspondence between both values - CD and grating depth.

Parameters are quite uniform over the whole range of 37 azimuthal angles, which leads to the conclusion that model describes very well the measured data. To be more quantitative, we estimated the errors of these parameters (with $95 \%$ confidence intervals) by equation (13) with $\sigma$ equal to the experimental RMS deviation between data and fits. The estimated error of the CD $(H)$ was $1.93 \mathrm{~nm}$, which is much larger than the fluctuations of this parameter with azimuthal angle: this unexpected constancy may come from the influence of the initial guess (which was kept constant at $250 \mathrm{~nm}$ ) on the final result of the Levenberg-Marquardt algorithm. Conversely, for the thickness $d$ the estimated error was $0.15 \mathrm{~nm}$, which is much smaller than the observed variations of $d$ with the azimuth, a more common behavior which can be due to small systematic errors and/or model inadequacies. 

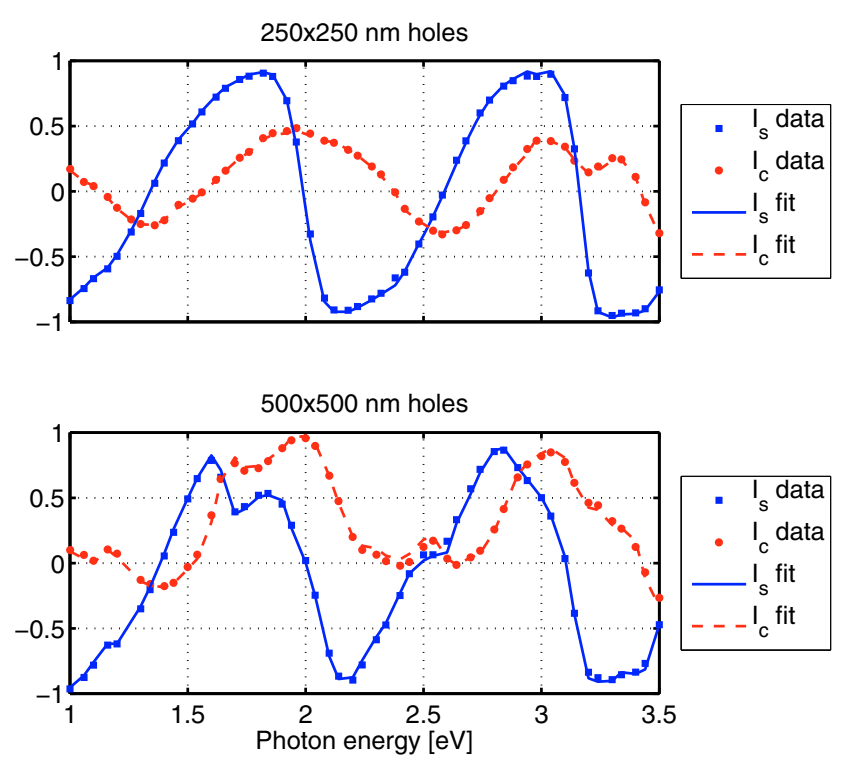

Fig. 7. (Color online) Fits of the ellipsometric parameters $I_{s}$ and $I_{c}$ of $250 \mathrm{~nm}$ (top) and $500 \mathrm{~nm}$ (bottom) holes in resist. Incidence angles were $70^{\circ}$ in both cases. Fitted values of CDs are 250.3 and $497.4 \mathrm{~nm}$ and depths are 393.5 and $406.1 \mathrm{~nm}$, respectively.

Typical values of the off-diagonal elements $B_{12}$ and $B_{21}$ of the Mueller matrix are very small, which means that this grating embodies only slight anisotropic behavior if it is rotated. This is obviously due to the high symmetry of the sample and small volume fill factor of holes $(1 / 16)$. Moreover, data are basically sensitive only to fill factor of the holes and it seems impossible to distinguish any other details about profile (e.g. round corners). Example of typical data and the fit can be found in Figure 9 for azimuthal angle $30^{\circ}$. Fit corresponds very well to data in all elements of normalized Mueller matrix, including offdiagonal ones with small values.

The results of Section 5 shows that data taken with the $500 \mathrm{~nm}$ holes sample is much richer, which motivated us to measure the sample at six different incidence angles: $45-70^{\circ}$. The results of fitting procedures can be seen on Figure 10, where the non-uniformity of fitted parameters can be observed. Note that every point in the plot corresponds to a measurement followed by a fit.

Regarding rectangular profile of the holes, there is necessary symmetry with respect to the zero angle and also $-90^{\circ}$ and $90^{\circ}$. Also, if profile is perfectly square, there are other symmetries with respect to the azimuthal angles $-45^{\circ}$ and $45^{\circ}$. Typical data and the fit can be found in Figure 11 at incidence angle of $45^{\circ}$ and azimuthal angle $30^{\circ}$.

The average estimated errors in the CD and the grating depth for the $500 \mathrm{~nm}$ holes grating at $45^{\circ}$ incidence are $1.14 \mathrm{~nm}$ and $0.28 \mathrm{~nm}$ within $95 \%$ confidence interval. In contrast with the $250 \mathrm{~nm}$ holes sample, these values are well below the observed variation of both $H$ and $d$ with the azimuth. Figure 10 shows this variation for all
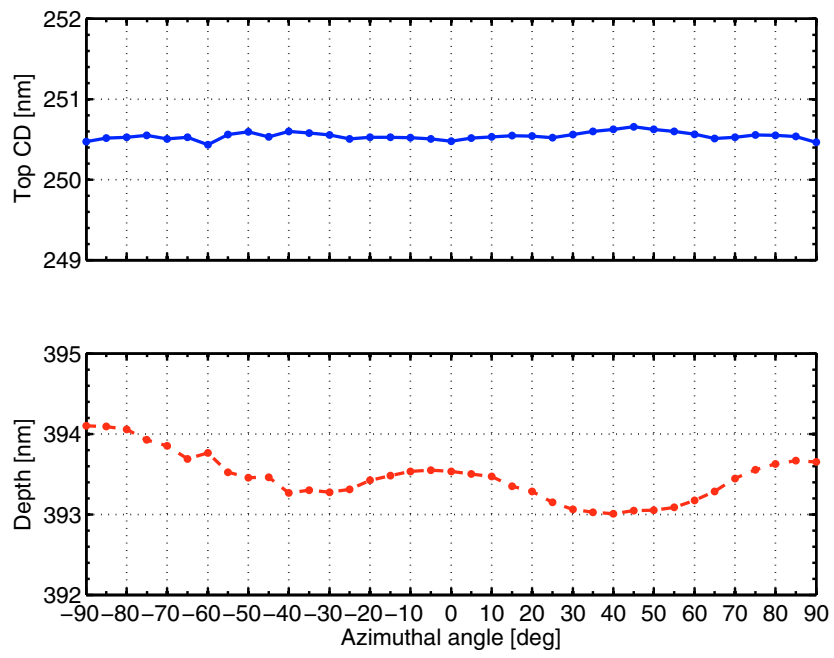

Fig. 8. (Color online) Results of fits of of $250 \mathrm{~nm}$ holes in resist. Incidence angle was $70^{\circ}$. Data for each azimuthal angle (with step of $5^{\circ}$ ) were fitted independently. Top part of figure shows fitted values of top $\mathrm{CD}$, while bottom part the grating depth. Units are in nanometers.
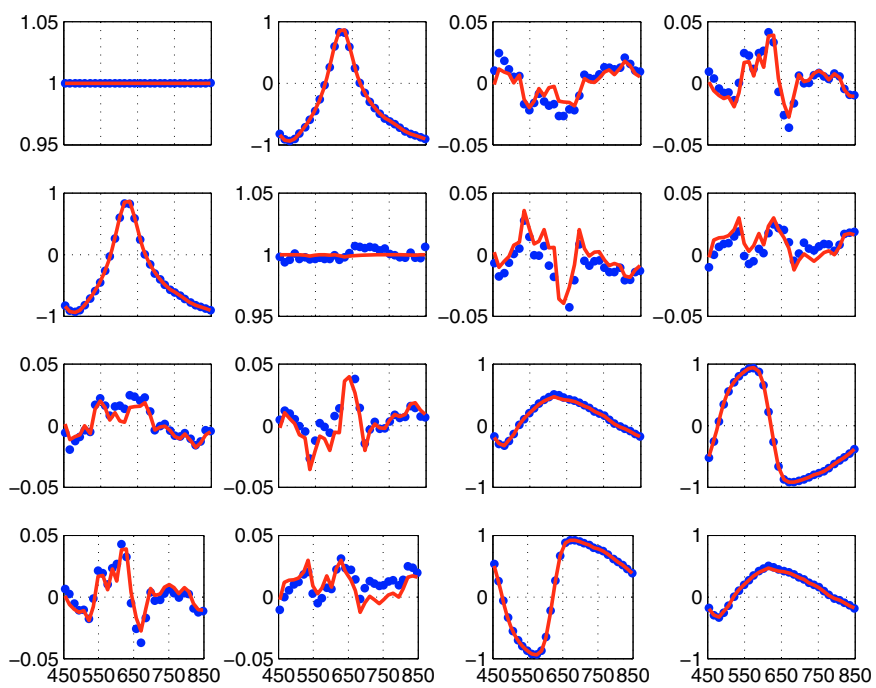

Fig. 9. (Color online) Example of the data (blue points) and fit (red lines) of the normalized Mueller matrices of the sample with $250 \mathrm{~nm}$ holes. Wavelengths are marked on $x$-axis, azimuthal angle in this case was $30^{\circ}$ and angle of incidence $70^{\circ}$.

investigated incidence angles. The fits at the smallest incidence angle of $45^{\circ}$ are the most uniform. From Figure 6 we know that the standard errors and correlation between parameters at $45^{\circ}$ are better than for the other angles. On the other hand, higher incidences feature decreased sensitivity and increased parameter correlations. Therefore, we assume that not only experimental errors are responsible for the non-uniformity of the fitted values, but also worse conditions of fits at higher incidence angles.

The residual differences between measured data and fit are plotted in Figure 12 for the $500 \mathrm{~nm}$ holes grating measured at $45^{\circ}$ incidence in the planar configuration $\left(0^{\circ}\right.$ azimuth). As the off-diagonal elements of 

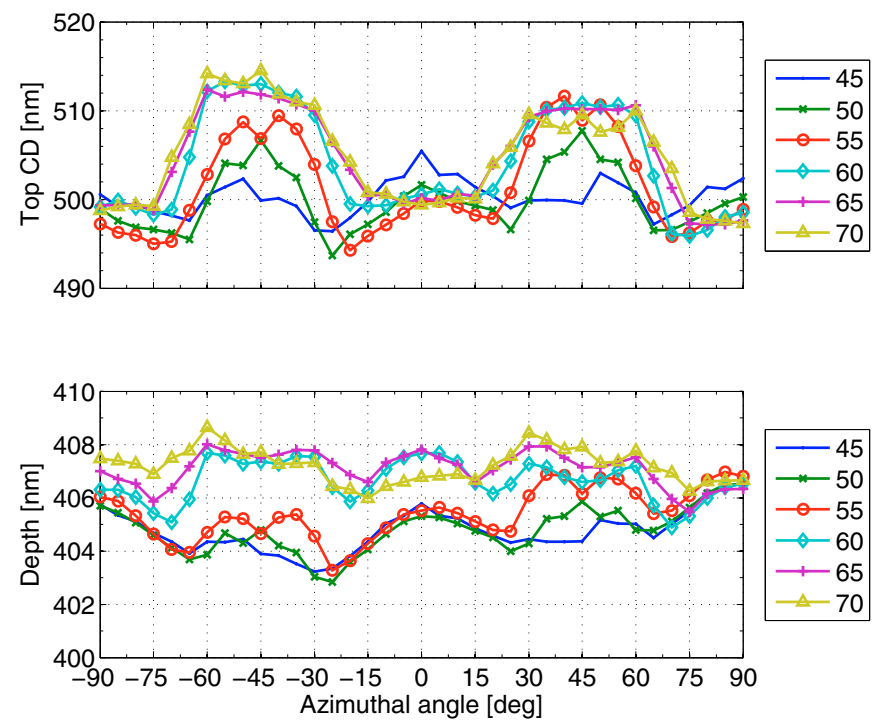

Fig. 10. (Color online) Fitted values of top CD and grating depth of the sample with $500 \mathrm{~nm}$ holes in nanometer scale. Curve correspond to the incidence angles marked on the right side of figure. Each point corresponds to one measurement and fit.
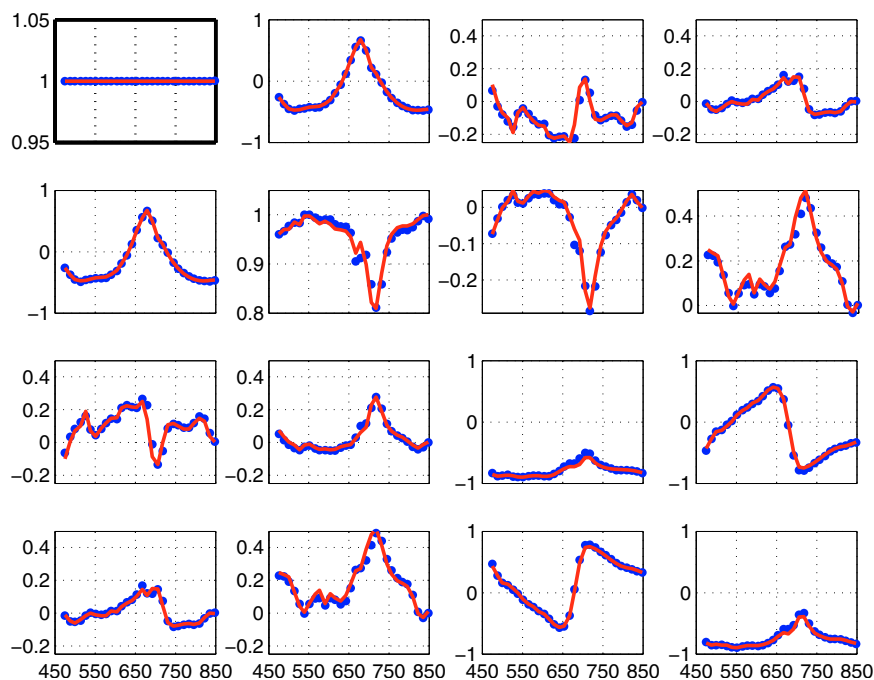

Fig. 11. (Color online) Example of the data (blue points) and fit (red lines) of the normalized Mueller matrices of the sample with $500 \mathrm{~nm}$ holes. Azimuthal angle is $30^{\circ}$ and angle of incidence is $45^{\circ}$.

normalized Mueller matrices are expected to vanish in this configuration, we can say that non-zero signals in offdiagonal elements are due to experimental errors, such as noise and systematic errors including azimuthal positioning. Full Mueller matrix measurements do provide some useful criteria to unveil such errors: for example the peaks observed in most elements around wavelengths of $675 \mathrm{~nm}$ involve a small depolarization of the emerging light, which is certainly due to measurement inaccuracies in this spectral region where the reflectivity drops to its lowest values, of the order of $2 \%$ and less. Moreover, we see on this figure that the residual differences between model and
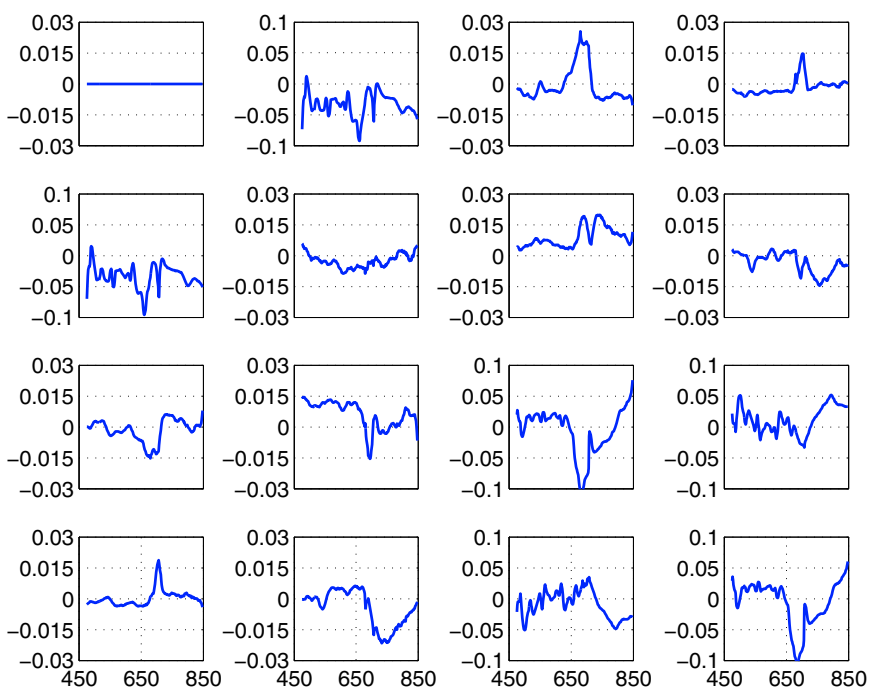

Fig. 12. (Color online) Residual difference between measured data and fit for $500 \mathrm{~nm}$ holes grating at $45^{\circ}$ incidence and $0^{\circ}$ azimuth. In this configuration the theoretical off-diagonal elements vanish.

measured data exhibit clear symmetry properties with respect to matrix transposition. These symmetries are obviously not compatible with the statistically independent noise properties assumed in Section 5, and are probably due to model inadequacies rather than measurement systematic errors, as such errors are not expected to preserve transposition symmetries with the calibration method used for the MM16. In any case, a rigorous treatment of such inadequacies is not available (one has only to change the model, if possible). However, the standard treatment implemented in Section 5 seems a quite reasonable first step to optimise the measurement configuration.

To compare virtual and real experiments, the correlations obtained from regression of measured data and "virtual experiments" were plotted in Figure 13b. Top part of the plot corresponds to the virtual experiment and bottom to the regression results. Very good correspondence is observed for all of the measured configurations. The values of the statistical errors are in the same manner compared in Figure 13c for hole width and Figure 13d for grating depth. The higher values from measurement regressions can be explained by the map of merit function plotted in Figure 13a, where for some angles the values are more than five times bigger than in virtual experiments. The observed values of the regression statistical errors in Figures $13 \mathrm{c}$ and $13 \mathrm{~d}$ are smaller than parameter dispersion in Figure 10, while inconsistent values appeared for the azimuthal angles with higher values of merit function in Figure 13a. At this moment, we can conclude that ideal model of experiment can be only a starting point to the determination of the optimal configuration, but it cannot fully replace actual measurement, which in general involves some systematic errors.

To increase sensitivity of parameters to the measured data, each azimuthal was fitted separately, but this time for all the incidence angles together. As this is very 


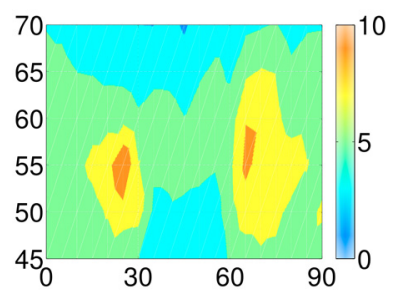

(a) Merit function

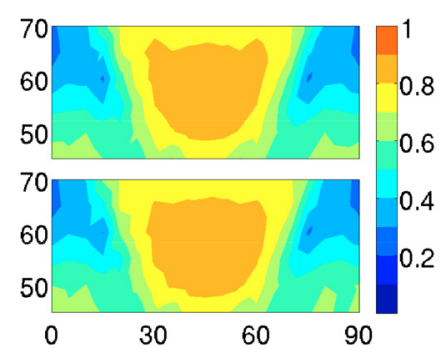

(b) Correlations (H-d)

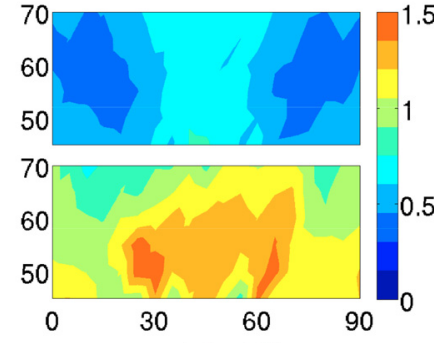

(c) $\Delta H$

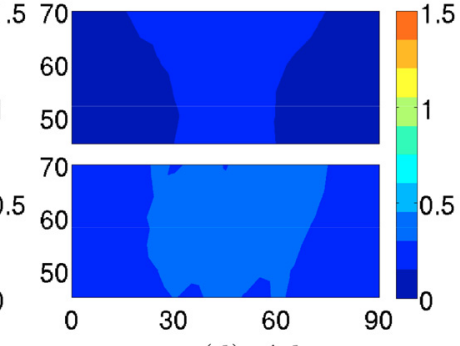

(d) $\Delta d$
Fig. 13. (Color online) Results of regression analysis of data measured on the sample with $500 \mathrm{~nm}$ holes. (a) Values of the merit function for the optimal values of parameters. (b), (c) and (d) Comparison of statistical uncertainties and correlations of the $H$ and $d$ parameters deduced from virtual (top plots) and actual experiments (bottom plots). Values are plotted for all measured incidence angles from $45^{\circ}$ to $70^{\circ}$ ( $y$-axis) and range $0-90^{\circ}$ of azimuthal angles ( $x$-axis).

time consuming procedure, only eight points with wavelengths from the range between 475 and 825 nm were chosen. To decrease calculation time, initial values for every azimuthal angle were taken from fitted values at previous angle, except the first one. This does not have high influence on the results - it was compared with fits from the constant initial values and no significant differences were observed. On the other hand, fitting started relatively close to minimum and total time was decreased by excluding some unnecessary iterations in the beginning.

Results of the fits in Figure 14 show much more uniform distribution of fitted values, which was expected from mixing multiple incidence angles data together. Errors in the $\mathrm{CD}$ and the grating depth are in the average $0.54 \mathrm{~nm}$ and $0.22 \mathrm{~nm}$ within the confidence interval of $95 \%$, which gives better confidence of the CD values than in the separate fits. The remaining non-uniformity of the fitted values is probably caused by experimental errors and also simplification of the model, which assumed square profile of the holes. From the electron microscope images in Figure 2 one can notice rounding corners of the holes. Some imperfections of the fits can be seen in Figure 15, which also indicates that the model does not perfectly correspond to the data. More complex profiles were not used in this work as this would have significantly increased calculation time, while at the end, simpler model provided sufficient accuracy. For the same reasons only eight spectral points
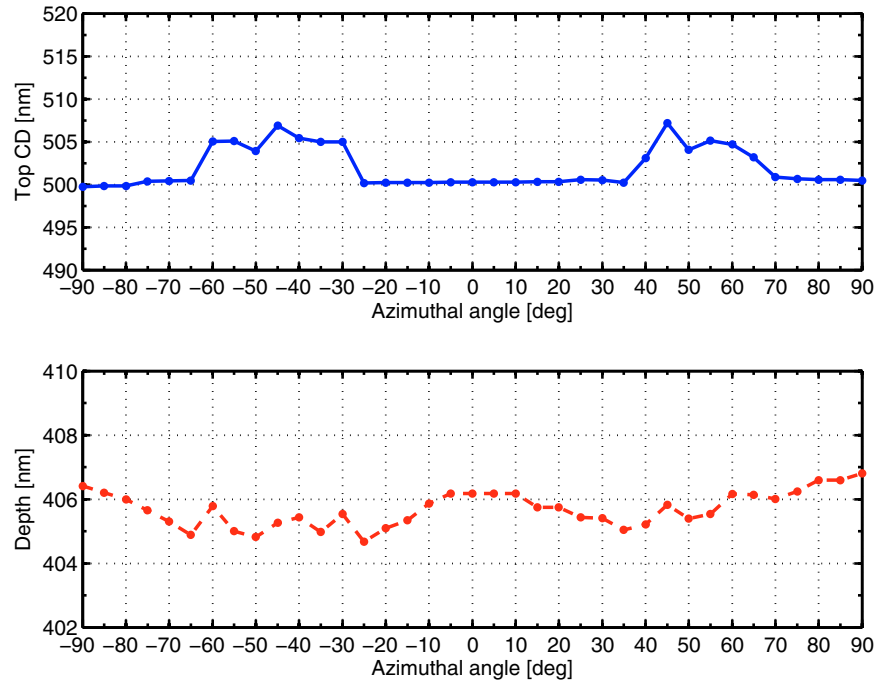

Fig. 14. (Color online) Fitted values of top CD and grating depth for the $500 \mathrm{~nm}$ holes sample. Data for incidence angles from $45^{\circ}$ to $70^{\circ}$ with step of $5^{\circ}$ were fitted simultaneously for each azimuthal angle.
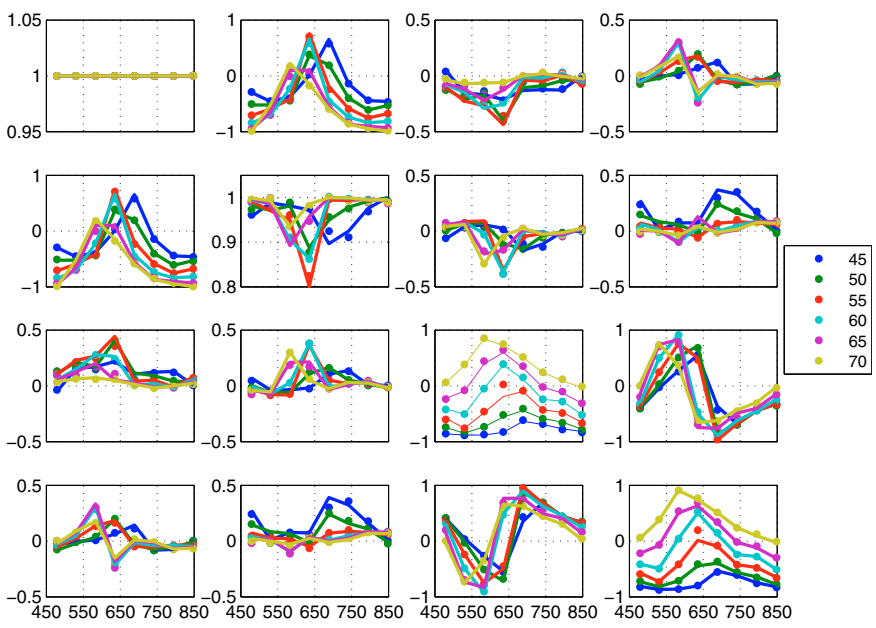

Fig. 15. (Color online) Example of the data (points) and fit (lines) of the normalized Mueller matrices of the sample with $500 \mathrm{~nm}$ holes at azimuthal angle $30^{\circ}$. Data for the incidence angles $45-70^{\circ}$ marked on the right side of plot were fitted together.

were used during fitting to decrease calculation times to a manageable amount.

\section{Conclusions}

Two samples with square arrays of square holes in a photoresist layer on silicon were characterized by spectral ellipsometry and Mueller matrix polarimetry. Both techniques gave close values of critical dimensions. Data from UVISEL provided more precise data in planar geometry due to wider spectral range, while MM16 shows higher robustness by enabling user to choose any experimental configuration. 
We showed that the sample with smaller holes exhibits very small in-plane anisotropy, which permits to determine only critical dimensions without any further information about profile. On contrary, sample with bigger holes shows slight non-uniformity of the critical dimensions, which gives a clear indication that more details about the profile can be in principle extracted from the data.

Comparison of the confidence intervals of fitted parameters has shown correspondence between theoretical "virtual experiments" and the measurements on the Mueller matrix polarimeter. This result shows that theoretical calculations can be used to estimate optimal measurement configuration in the cases where the overall measurement precision is at least approximately known.

We have demonstrated that theoretical study to estimate optimal measurement configuration is very useful, sometimes even unavoidable, to get precise and reliable results from a single measurement. Statistical quantities including parameter errors and correlation coefficient were compared between "virtual experiment" and measurement regression, showing excellent agreement between parameter correlations. Dispersion of the optimal values for different azimuthal angles is higher than statistical errors obtained from regressions, which means that systematic errors and and/or model inadequacies played crucial role in the final precision of the regression. Therefore, the typical sensitivity calculations based on virtual experiments and a model a priori assumed to be ideal provide a good starting point to the determination of the optimal configuration, but it cannot fully replace actual measurement.

\section{References}

1. B.J. Rice, H. Cao, M. Grumski, J. Roberts, Microelectr. Eng. 83, 1023 (2006)

2. T. Novikova, A. De Martino, P. Bulkin, Q. Nguyen, B. Drévillon, V. Popov, A. Chumakov, Opt. Expr. 15, 2033 (2007)

3. H.T. Huang, F.L. Terry Jr., Thin Solid Films 468, 339 (2004)

4. H.T. Huang, W. Kong, F.L. Terry Jr., Appl. Phys. Lett. $\mathbf{7 8}, 3983$ (2001)

5. T. Novikova, A. De Martino, S.B. Hatit, B. Drévillon, Appl. Opt. 45, 3688 (2006)

6. R.M.A. Azzam, N.M. Bashara, Ellipsometry and Polarized Light, 2nd edn. (North-Holland, Amsterdam, 1987)

7. E. Compain, S. Poirier, B. Drévillon, Appl. Opt. 38, 3490 (1999)

8. J. Tyo, Opt. Lett. 25, 1198 (2000)

9. E. Garcia-Caurel, A. De Martino, B. Drévillon, Thin Solid Films 455-456, 120 (2004)

10. Handbook of Optical Constants of Solids I and II and III, edited by E.D. Palik (Academic Press, 1991)

11. G.E. Jellison Jr., F.A. Modine, Appl. Phys. Lett. 69, 371 (1996)

12. M.G. Moharam, T.K. Gaylord, J. Opt. Soc. Am. 72, 1385 (1982)

13. L. Li, J. Opt. Soc. Am. A 13, 1870 (1996)

14. L. Li, J. Opt. Soc. Am. A 13, 1024 (1996)

15. W.H. Press, S.A. Teukolsky, W.T. Vetterling, B.P. Flannery, Numerical Recipes in $\mathrm{C}++$. The art of scientifique computing, 2nd edn. (Cambridge, 2002) 